\title{
Existence of solutions of elliptic boundary value problems with mixed type nonlinearities
}

Anmin Mao*, Yan Zhu and Shixia Luan

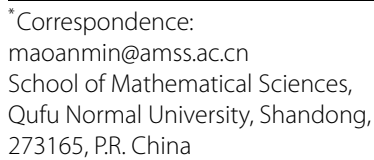

\begin{abstract}
We study the existence of a nontrivial solution of the following elliptic boundary value problem with mixed type nonlinearities:

$$
\begin{cases}-\Delta u=f(x, u) & \text { in } \Omega, \\ u=0 & \text { on } \partial \Omega\end{cases}
$$

where $f(x, u)=-K_{u}+W_{u}$. We consider the problem in a different case:

$\lim _{|u| \rightarrow \infty} f(x, u) / u=\infty, \lim _{|u| \rightarrow 0} f(x, u) / u$ is some constant. Assuming that $K$ satisfies the "pinching" condition, and $W$ satisfies a more general superquadratic growth condition than the well-known Ambrosetti-Rabinowitz condition usually used in literature, we obtain a nontrivial solution via the Mountain Pass Lemma.
\end{abstract}

MSC: $35 \mathrm{~J} 65 ; 35 \mathrm{~J} 20 ; 47 \mathrm{J10}$

Keywords: pinching condition; Mountain Pass Lemma; Cerami condition

\section{Introduction}

In this paper, we shall be concerned with the elliptic boundary value problem in a different case

$$
\begin{cases}-\Delta u=f(x, u) & \text { in } \Omega, \\ u=0 & \text { on } \partial \Omega,\end{cases}
$$

where $\Omega \subset R^{N}(N>2)$ is a bounded open domain with a smooth boundary $\partial \Omega$ and $f \in$ $C\left(\Omega \times R^{1}, R^{1}\right)$.

The existence of nontrivial weak solutions for $(\mathrm{P})$ have been studied in many papers, see [1-12]. Su and Zhao in [2] considered problem (P) for resonance case at infinity, $\lim _{|u| \rightarrow \infty} \frac{f(x, u)}{u}=\lambda_{k}$, where $\lambda_{k}$ is an eigenvalue of the linear boundary value problem

$$
\begin{cases}-\Delta u=\lambda u & \text { in } \Omega, \\ u=0 & \text { on } \partial \Omega,\end{cases}
$$


the existence of multiple nontrivial solutions for $(\mathrm{P})$ are obtained by minimax methods and Morse theory. Ambrosetti and Rabinowitz in [3] established the existence of a nontrivial solution for problem $(\mathrm{P})$ by assuming the following conditions:

$\left(f_{1}^{\prime}\right) f(x, 0)=0, \lim _{u \rightarrow 0} \frac{f(x, u)}{u}=0$, uniformly in a.e. $x \in \Omega$.

$\left(f_{2}^{\prime}\right)$ There exist two positive constants $a$ and $b$ such that

$$
|f(x, u)| \leq a+b|u|^{p} \quad \text { for some } 0 \leq p<\frac{N+2}{N-2}, \forall u \in R^{1}, x \in \Omega .
$$

And the following well-known Ambrosetti-Rabinowitz condition ((AR) for short):

$$
\exists \theta>2, R_{0}>0 \quad \text { s.t. } 0<\theta F(x, u) \leq u f(x, u), \quad \text { for all }|u| \geq R_{0}, x \in \Omega \text {, }
$$

where $F(x, u)=\int_{0}^{u} f(x, s) d s$.

Since then, the (AR) condition has been used extensively in many literature sources, see [12-18]. It is well known that the (AR) condition is quite natural and convenient not only to ensure that the Euler-Lagrange functional associated to problem (P) has a mountain pass geometry but also to guarantee that the Palais-Smale sequence of the Euler-Lagrange functional is bounded. Let $E$ be a Hilbert space and $G \in C^{1}\left(E, R^{1}\right)$. Recall that the sequence $\left\{u_{n}\right\}_{n \in N} \subset E$ is said to be a Palais-Smale sequence of $G$ provided that $\left\{G\left(u_{n}\right)\right\}$ is bounded and $G^{\prime}\left(u_{n}\right) \rightarrow 0$ as $n \rightarrow+\infty$, the function $G$ satisfies the Palais-Smale condition ((PS) for short) if and only if any Palais-Smale sequence for $G$ contains a convergent subsequence. The function $G$ satisfies the Cerami condition $\left((C)\right.$ for short) if any sequence $\left\{u_{n}\right\}_{n \in N}$ in $E$ satisfying $G\left(u_{n}\right)$ is bounded and $G^{\prime}\left(u_{n}\right)\left(1+\left\|u_{n}\right\|\right) \rightarrow 0$ as $n \rightarrow+\infty$ has a convergent subsequence.

Without (AR), it becomes more complicated. Indeed, there are many functions which are superlinear, but it is not necessary to satisfy (AR) even if $1<\theta \leq 2$. Willem and Zou stated the following examples:

$$
f(x, u)=\mu|u|^{\mu-2} u+(\mu-1)|u|^{\mu-3} u \sin ^{2} u+|u|^{\mu-1} \sin 2 u, \quad u \in R^{1} \backslash\{0\},
$$

where $\mu>2$. Then it is easy to check that (AR) does not hold even for any $\theta>\mu-1>1$. On the other hand, in order to verify (AR), it usually is an annoying task to compute a primitive function of $f$ and sometimes it is almost impossible. For example,

$$
f(x, u)=|u| u\left(1+e^{(1+|\sin u|)^{\alpha}}+|\cos u|^{\alpha}\right), \quad u \in R^{1},
$$

where $\alpha>0$.

Some authors have tried to drop or weaken the above superlinear condition (AR) in recent years, see $[4-8,11,19]$. Miyagaki and Souto [8] adapted some monotonicity arguments studying the existence of nontrivial weak solutions of $(\mathrm{P})$.

The aim of the manuscript is to consider the problem in a different case: $\lim _{|u| \rightarrow \infty} f(x, u) / u=\infty, \lim _{|u| \rightarrow 0} f(x, u) / u$ is some constant. We study this problem under "pinching" condition and the general superquadratic condition. The case that $F(x, u)$ has a part with "pinching" condition has been considered only by few authors, see [13, 20]. Since $F(x, u)$ does not satisfy the $\left(f^{\prime}\right)$ and (AR), problem (P) becomes more delicate. The 
main difficulty when dealing with this problem is the lack of compactness of the Sobolev embedding theorem.

In this paper, here, $F(t, u):=\int_{0}^{u} f(t, s) d s$ replaced by $-K+W$, satisfy

$\left(F_{1}\right) F(x, u)=-K(x, u)+W(x, u), K, W: \Omega \times R^{1} \rightarrow R^{1}$ are $C^{1}$-maps.

$\left(K_{1}\right)$ There are two positive constants $b_{1}$ and $b_{2}$ such that

$$
b_{1}|u|^{2} \leq K(x, u) \leq b_{2}|u|^{2}, \quad \text { for all }(x, u) \in \Omega \times R^{1} .
$$

$\left(K_{2}\right)$ There exists $\varrho \in(1,2]$ such that

$$
K(x, u) \leq K_{u}(x, u) u \leq \varrho K(x, u), \quad \text { for all }(x, u) \in \Omega \times R^{1} .
$$

$\left(W_{1}\right) \quad W(x, u) \geq 0$ and $W_{u}(x, u)=o(|u|)$ as $|u| \rightarrow 0$ uniformly in $x$.

$\left(W_{2}\right) W(x, u) / u^{2} \rightarrow \infty$ as $|u| \rightarrow \infty$ uniformly in $x$.

$\left(W_{3}\right)$ Set $\tilde{W}(x, u):=\frac{1}{2} W_{u}(x, u) u-W(x, u), \tilde{W}(x, u)>0$ if $u \neq 0, \tilde{W}(x, u) \rightarrow \infty$ as $|u| \rightarrow$ $\infty$ uniformly in $x$, and there exist $r_{0}>0$ and $\sigma>N / 2$ such that $\left|W_{u}(x, u)\right|^{\sigma} \leq$ $c_{0} \tilde{W}(x, u)|u|^{\sigma}$ if $|u| \geq r_{0}$.

We will prove the following results.

Theorem 1.1 If assumptions $\left(F_{1}\right),\left(K_{1}\right),\left(K_{2}\right)$ and $\left(W_{1}\right)-\left(W_{3}\right)$ are satisfied, then problem $(P)$ has a nontrivial weak solution.

\section{Remark 1}

(i) Our assumptions $\left(W_{2}\right),\left(W_{3}\right)$ are weaker than (AR), and there is no monotone condition;

(ii) The condition $\left(K_{2}\right)$ can be written in the form $1 \leq \frac{K_{u}(x, u) u}{K(x, u)} \leq \varrho, \varrho \in(1,2]$ which is weaker than the condition $1 \leq \frac{K_{u}(x, u) u}{K(x, u)} \leq 2$ in $[13,20]$.

Example 1 Consider the functions

$$
K(x, u)=[1+\exp (-|x|)] u^{2}, \quad W(x, u)=\left(2-\frac{1}{1+|x|}\right) u^{2} \ln \left(1+u^{2}\right) .
$$

A straightforward computation shows that $K$ and $W(x, u)$ satisfy the assumptions of Theorem 1.1, but neither $F(x, u)$ nor $W(x, u)$ satisfy the (AR) condition.

Example 2 Consider the more general functions

$$
K_{u}(x, u)=V(x) u, \quad W_{u}(x, u)=g(x, u)
$$

where $g(x, u)$ is of superlinear growth as $|u| \rightarrow \infty$. A straightforward computation shows that $K$ and $W$ satisfy the assumptions of Theorem 1.1.

We will prove that the function associated with $(\mathrm{P})$ has Mountain Pass geometry and satisfies the $(C)$ condition. The remainder of the paper is organized as follows. In Section 2, we deal with the variational setting. In Section 3, we give the details of the proof of Theorem 1.1. 


\section{Preliminary results}

Let $H:=H_{0}^{1}(\Omega)$ be the Sobolev space equipped with the inner product and the norm

$$
(u, v)=\int_{\Omega}(\nabla u \cdot \nabla v+u v) d x, \quad\|u\|=(u, u)^{\frac{1}{2}}, \quad u, v \in H
$$

And we denote the usual $L^{p}(\Omega)$-norm

$$
\|u\|_{p}=\left(\int_{\Omega}|u(x)|^{p} d x\right)^{\frac{1}{p}} .
$$

Our approach will be the variational techniques. Define the Euler-Lagrange functional associated to problem $(\mathrm{P})$ given by

$$
\Phi(u)=\frac{1}{2} \int_{\Omega}|\nabla u|^{2} d x-\int_{\Omega}[-K(x, u)+W(x, u)] d x, \quad \text { for all } u \in H .
$$

From the assumptions on $f$, it is standard to check that $\Phi \in C^{1}$ whose Gateaux derivative is

$$
\Phi^{\prime}(u) v=\int_{\Omega} \nabla u \cdot \nabla v d x-\int_{\Omega}\left[-K_{u}(x, u) v+W_{u}(x, u) v\right] d x, \quad \text { for all } u, v \in H .
$$

Let $\eta: H \rightarrow[0,+\infty)$ be given by

$$
\eta(u):=\left(\int_{\Omega}\left[|\nabla u|^{2}+2 K(x, u)\right] d x\right)^{\frac{1}{2}}
$$

Hence

$$
\Phi(u)=\frac{1}{2} \eta^{2}(u)-\int_{\Omega} W(x, u) d x .
$$

By $\left(K_{2}\right)$,

$$
\Phi^{\prime}(u) u \leq \int_{\Omega}|\nabla u|^{2} d x+\int_{\Omega} \varrho K(x, u) d x-\int_{\Omega} W_{u}(x, u) u d x .
$$

By $\left(K_{1}\right)$ and set $b_{1,1}:=\min \left\{1,2 b_{1}\right\}, b_{2,2}:=\max \left\{2,2 b_{2}\right\}$,

$$
b_{1,1}\|u\|^{2} \leq \eta(u)^{2} \leq b_{2,2}\|u\|^{2} .
$$

It is worth pointing out that if the function $K(x, u)$ is of the form $\frac{1}{2} V(x) u^{2}$ with $V(x) \in$ $C^{1}\left(\Omega, R^{1}\right)$ and $\inf _{\Omega} V(x) \geq V_{0}>0$ then $\eta$ in a Hilbert space $X=\left\{u \in H_{0}^{1}(\Omega) ; \int_{\Omega} V(x) u^{2}<\infty\right\}$ is equivalent to the norm $\|\cdot\|$; however, if the function $K(x, u)$ is not of the form $\frac{1}{2} V(x) u^{2}$, $\eta$ is not a norm because of the lack of norm's linear property.

Lemma 2.1 (see [5]) Let $H$ be a real Banach space, $\Phi \in C^{1}(H, R)$, satisfying $\Phi(0)=0$. Moreover,

(i) there exist $\rho, \alpha>0$ such that $\Phi_{\mid \partial B_{\rho}(0)} \geq \alpha$, 
(ii) there exists $e \in H \backslash \overline{B_{\rho}(0)}$ such that $\Phi(e) \leq 0$.

Then there exists a sequence $\left\{u_{n}\right\} \in H$ such that $\left\|\Phi^{\prime}\left(u_{n}\right)\right\|\left(1+\left\|u_{n}\right\|\right) \rightarrow 0$ and $\Phi\left(u_{n}\right) \rightarrow c \geq \alpha$ as $n \rightarrow \infty$.

Lemma 2.2 (see [1]) Assume that $|\Omega|<\infty, 1 \leq p, r<\infty, f \in C(\bar{\Omega} \times R)$ and $|f(x, u)| \leq$ $c\left(1+|u|^{\frac{p}{r}}\right)$. Then, for every $u \in L^{p}(\Omega), f(x, u) \in L^{r}(\Omega)$ and the operator $A: L^{p}(\Omega) \rightarrow L^{r}(\Omega)$, $u \mapsto f(x, u)$ is continuous.

\section{Proofs of theorems}

First of all, we recall a property of the function $K(x, u)$, which is necessary to the proof of the geometric structure of the $C^{1}$ functional $\Phi$.

Fact 1 Assume that $\left(K_{2}\right)$ holds, then

$$
K(x, u) \leq K\left(x, \frac{u}{|u|}\right)|u|^{\varrho}, \quad \text { for all } x \in \Omega \text { and }|u| \geq 1
$$

Proof Define $G: s \rightarrow K\left(x, s^{-1} u\right) s^{\varrho}, s \in(0,+\infty)$

$$
\begin{aligned}
G^{\prime}(s) & =-K_{u}\left(x, s^{-1} u\right) \frac{u}{s^{2}} s^{\varrho}+K\left(x, s^{-1} u\right) \varrho s^{\varrho-1} \\
& =-K_{u}\left(x, s^{-1} u\right) s^{-1} u s^{\varrho-1}+K\left(x, s^{-1} u\right) \varrho s^{\varrho-1} \\
& =s^{\varrho-1}\left[-K_{u}\left(x, s^{-1} u\right) s^{-1} u+K\left(x, s^{-1} u\right) \varrho\right] .
\end{aligned}
$$

By $\left(K_{2}\right), G^{\prime}(s) \geq 0$, which implies $G(s)$ is non-decreasing. So, we have

$$
K(x, u)=G(1) \leq G(s)=K\left(x, \frac{u}{|u|}\right)|u|^{\varrho}, \quad \text { if }|u|=s \geq 1
$$

Next we discuss the geometric structure of the $C^{1}$ functional $\Phi$ on $H$.

Lemma 3.1 Under the assumptions of Theorem 1.1, there are constants $\rho, \alpha>0$ such that $\Phi_{\mid \partial B_{\rho}(0)} \geq \alpha$.

Proof From $\left(W_{1}\right)$ and $\left(W_{3}\right)$, as $|u|>r_{0}$, we have

$$
\begin{aligned}
\left|W_{u}(x, u)\right|^{\sigma} & \leq c_{0}\left(\frac{1}{2} W_{u}(x, u) u-W(x, u)\right)|u|^{\sigma} \\
& \leq c_{1} W_{u}(x, u)|u|^{\sigma+1},
\end{aligned}
$$

where $c_{1}$ is a positive constant. Hence as $|u|>r_{0}$,

$$
\left|W_{u}(x, u)\right| \leq c_{1}|u|^{\sigma+1 /(\sigma-1)}
$$

From $\sigma>N / 2$, we know $\sigma+1 /(\sigma-1)<2^{*}-1$, so we can choose $2 \sigma /(\sigma-1) \leq p<2^{*}$. And using ( $\left.W_{1}\right)$ again, we observe that for any given $\epsilon>0$ there is $c_{\epsilon}>0$ such that

$$
\left|W_{u}(x, u)\right| \leq \epsilon|u|+c_{\epsilon}|u|^{p-1}
$$


and

$$
|W(x, u)| \leq \epsilon|u|^{2}+c_{\epsilon}|u|^{p}
$$

It follows from (3.2) and the Sobolev embedding theorem that for all $u \in H$

$$
\int_{\Omega} W(x, u) d x \leq \epsilon\|u\|_{2}^{2}+c_{\epsilon}\|u\|_{p}^{p} \leq \epsilon\|u\|_{2}^{2}+c_{\epsilon} c\|u\|^{p}
$$

where $c$ is a positive constant. Then combining $\left(K_{1}\right)$ and (3.3), we obtain

$$
\begin{aligned}
\Phi(u) & \geq \frac{1}{2} \int_{\Omega}|\nabla u|^{2} d x+b_{1}\|u\|_{2}^{2}-\left(\epsilon\|u\|_{2}^{2}+c_{\epsilon} c\|u\|^{p}\right) \\
& =\frac{1}{2} \int_{\Omega}|\nabla u|^{2} d x+\left(b_{1}-\epsilon\right)\|u\|_{2}^{2}-c_{\epsilon} c\|u\|^{p} \geq \min \left\{\frac{1}{2},\left(b_{1}-\epsilon\right)\right\}\|u\|^{2}-c_{\epsilon} c\|u\|^{p}
\end{aligned}
$$

set $b_{0}=\min \left\{\frac{1}{2},\left(b_{1}-\epsilon\right)\right\}$, it is clear that $b_{0}>0$. We choose $\|u\|=\rho=\left(\frac{\frac{1}{2} b_{0}}{c_{\epsilon} c}\right)^{\frac{1}{p-2}}$ and $\alpha=$ $\frac{1}{2} \rho^{2} b_{0}$, then

$$
\Phi(u) \geq \alpha
$$

Lemma 3.2 Under the assumptions of Theorem 1.1, there exists $e \in H \backslash \overline{B_{\rho}(0)}$ such that $\Phi(e)<0$.

Proof Let $e_{0} \in H \backslash 0, M=\max _{x \in \Omega,|u| \leq 1} K(x, u)$ and $A>\frac{(2 M+1)\left\|e_{0}\right\|^{2}}{2\left\|e_{0}\right\|_{2}^{2}}$. By $\left(W_{2}\right)$, there exists $B>0$ such that

$$
W(x, u) \geq A|u|^{2}-B, \quad \text { for all } x \in \Omega, u \in H
$$

As $\varrho<2$, by Fact 1 , we have

$$
\begin{aligned}
\eta^{2}\left(\xi e_{0}\right) & =\int_{\Omega}\left[\left|\nabla \xi e_{0}\right|^{2}+2 K\left(x, \xi e_{0}\right)\right] d x \\
& \leq \int_{\Omega}\left|\nabla e_{0}\right|^{2} \xi^{2} d x+2 \int_{\left\{x \in \Omega ;\left|\xi e_{0}\right| \leq 1\right\}} K\left(x, \xi e_{0}\right) d x+2 \int_{\left\{x \in \Omega ;\left|\xi e_{0}\right| \geq 1\right\}} K\left(x, \xi e_{0}\right) d x \\
& \leq \int_{\Omega}\left|\nabla e_{0}\right|^{2} \xi^{2} d x+2 M|\Omega|+2 M \int_{\left\{x \in \Omega ;\left|\xi e_{0}\right| \geq 1\right\}}\left|\xi e_{0}\right|^{\varrho} d x \\
& \leq \int_{\Omega}\left|\nabla e_{0}\right|^{2} \xi^{2} d x+2 M|\Omega|+2 M \int_{\left\{x \in \Omega ;\left|\xi e_{0}\right| \geq 1\right\}}\left|\xi e_{0}\right|^{2} d x \\
& \leq \xi^{2}(1+2 M)\left\|e_{0}\right\|^{2}+2 M|\Omega| .
\end{aligned}
$$

Then, by inequalities (3.4) and (3.5), we get

$$
\begin{aligned}
\Phi\left(\xi e_{o}\right) & =\frac{1}{2} \eta^{2}\left(\xi e_{0}\right)-\int_{\Omega} W\left(x, \xi e_{0}\right) d x \leq \frac{1+2 M}{2} \xi^{2}\left\|e_{0}\right\|^{2}+M|\Omega|-A \xi^{2}\left\|e_{0}\right\|_{2}^{2}-B|\Omega| \\
& =\left(\frac{1+2 M}{2}\left\|e_{0}\right\|^{2}-A\left\|e_{0}\right\|_{2}^{2}\right) \xi^{2}+(M-B)|\Omega| .
\end{aligned}
$$


By the choice of $A$, we have $\left(\frac{1+2 M}{2}\left\|e_{0}\right\|^{2}-A\left\|e_{0}\right\|_{2}^{2}\right)<0$, so there exists $\xi_{0} \in R^{1}$ such that if $e=\xi_{0} e_{0}$, then

$$
\Phi(e)<0 .
$$

Suppose that the assumptions of Theorem 1.1 hold, we have Lemma 3.1 and Lemma 3.2. Now it follows from Lemma 2.1 that there is a sequence $\left\{u_{n}\right\} \subset H$ such that

$$
\left\|\Phi^{\prime}\left(u_{n}\right)\right\|\left(1+\left\|u_{n}\right\|\right) \rightarrow 0 \quad \text { and } \quad \Phi\left(u_{n}\right) \rightarrow c \geq \alpha \quad \text { as } n \rightarrow \infty .
$$

Lemma 3.3 Under the assumptions of Theorem 1.1, the functional $\Phi$ satisfies the $(C)$ condition.

Proof Let $\left\{u_{n}\right\} \subset H$ be such that

$$
\Phi\left(u_{n}\right) \text { is bounded and }\left(1+\left\|u_{n}\right\|\right) \Phi^{\prime}\left(u_{n}\right) \rightarrow 0 .
$$

By $\left(K_{2}\right)$ we observe that for large $n$,

$$
\begin{aligned}
c_{2} & \geq \Phi\left(u_{n}\right)-\frac{1}{2} \Phi^{\prime}\left(u_{n}\right) u_{n} \\
& =\int_{\Omega} K\left(x, u_{n}\right) d x-\int_{\Omega} \frac{1}{2} K_{u}\left(x, u_{n}\right) u_{n} d x+\int_{\Omega} \frac{1}{2} W_{u}\left(x, u_{n}\right) u_{n}-W\left(x, u_{n}\right) d x \\
& \geq \int_{\Omega} K\left(x, u_{n}\right)-\frac{\varrho}{2} K\left(x, u_{n}\right) d x+\int_{\Omega} \frac{1}{2} W_{u}\left(x, u_{n}\right) u_{n}-W\left(x, u_{n}\right) d x \\
& \geq \int_{\Omega} \tilde{W}\left(x, u_{n}\right) d x .
\end{aligned}
$$

Arguing indirectly, assume as a contradiction that $\left\|u_{n}\right\| \rightarrow \infty$. Setting $v_{n}=u_{n} /\left\|u_{n}\right\|$, then $\left\|v_{n}\right\|=1$ and since the embedding $H \hookrightarrow L^{s}$ for $s \in\left[2,2^{*}\right)$, we have $\left\|v_{n}\right\|_{s} \leq \gamma_{s}\left\|v_{n}\right\|=\gamma_{s}$. Observe that, from (3.8), $\left(K_{1}\right)$ and $\left(K_{2}\right)$

$$
\begin{aligned}
\Phi^{\prime}\left(u_{n}\right) u_{n} & =\int_{\Omega}\left|\nabla u_{n}\right|^{2} d x+\int_{\Omega} K_{u}\left(x, u_{n}\right) u_{n} d x-\int_{\Omega} W_{u}\left(x, u_{n}\right) u_{n} d x \\
& \geq \int_{\Omega}\left|\nabla u_{n}\right|^{2} d x+\int_{\Omega} K\left(x, u_{n}\right) d x-\int_{\Omega} W_{u}\left(x, u_{n}\right) u_{n} d x \\
& \geq \int_{\Omega}\left|\nabla u_{n}\right|^{2} d x+\int_{\Omega} b_{1}\left|u_{n}\right|^{2} d x-\int_{\Omega} W_{u}\left(x, u_{n}\right) u_{n} d x \\
& \geq \int_{\Omega} \frac{1}{2}\left|\nabla u_{n}\right|^{2} d x+\int_{\Omega} b_{1}\left|u_{n}\right|^{2} d x-\int_{\Omega} W_{u}\left(x, u_{n}\right) u_{n} d x \\
& \geq\left\|u_{n}\right\|^{2}\left(\frac{1}{2} b_{1,1}-\int_{\Omega} \frac{W_{u}\left(x, u_{n}\right) v_{n}}{\left\|u_{n}\right\|} d x\right) .
\end{aligned}
$$

It follows that for any $\epsilon>0$ and $n$ large enough,

$$
\int_{\Omega} \frac{W_{u}\left(x, u_{n}\right) v_{n}}{\left\|u_{n}\right\|} d x \geq \frac{b_{1,1}}{2}-\epsilon
$$


Set for $r \geq 0$

$$
h(r):=\inf \left\{\tilde{W}(x, u): x \in \Omega \text { and } u \in R^{1} \text { with }|u| \geq r\right\} .
$$

By $\left(W_{3}\right), h(r)>0$ for all $r>0$ and $h(r) \rightarrow \infty$ as $r \rightarrow \infty$. For $0 \leq a<b$, let

$$
\Omega_{n}(a, b)=\left\{x \in \Omega: a \leq\left|u_{n}(x)\right|<b\right\}
$$

and

$$
C_{a}^{b}=\inf \left\{\frac{\tilde{W}(x, u)}{u^{2}} \mid x \in \Omega \text { and } u \in R^{1} \text { with } a \leq\left|u_{n}(x)\right|<b\right\} .
$$

Since $\tilde{W}(x, u)>0$ if $u \neq 0$, one has $C_{a}^{b}>0$ and

$$
\tilde{W}\left(x, u_{n}(x)\right) \geq C_{a}^{b}\left|u_{n}(x)\right|^{2} \quad \text { for all } x \in \Omega_{n}(a, b) .
$$

It follows from (3.9) that

$$
\begin{aligned}
c_{2} & \geq \int_{\Omega} \tilde{W}\left(x, u_{n}\right) d x \\
& =\int_{\Omega_{n}(0, a)} \tilde{W}\left(x, u_{n}\right) d x+\int_{\Omega_{n}(a, b)} \tilde{W}\left(x, u_{n}\right) d x+\int_{\Omega_{n}(b, \infty)} \tilde{W}\left(x, u_{n}\right) d x \\
& \geq \int_{\Omega_{n}(0, a)} \tilde{W}\left(x, u_{n}\right) d x+C_{a}^{b} \int_{\Omega_{n}(a, b)}\left|u_{n}(x)\right|^{2} d x+h(b)\left|\Omega_{n}(b, \infty)\right| .
\end{aligned}
$$

Set $\tau:=2 \sigma /(\sigma-1)$, since $\sigma>N / 2$, one sees $\tau \in\left(2,2^{2}\right)$. Fix arbitrarily $\hat{\tau} \in\left(\tau, 2^{\prime \prime}\right)$, using (3.11),

$$
\left|\Omega_{n}(b, \infty)\right| \leq \frac{c_{2}}{h(b)} \rightarrow 0 \quad \text { as } b \rightarrow \infty \text { uniformly in } n,
$$

which implies by the Hölder inequality that

$$
\begin{aligned}
\int_{\Omega_{n}(b, \infty)}\left|v_{n}\right|^{\tau} d x & \leq\left(\int_{\Omega_{n}(b, \infty)} 1 d x\right)^{1-\frac{\tau}{\hat{\tau}}}\left(\int_{\Omega_{n}(b, \infty)}\left|v_{n}\right|^{\tau \frac{\hat{\tau}}{\tau}} d x\right)^{\frac{\tau}{\hat{\tau}}} \\
& \leq \gamma_{\hat{\tau}}^{\tau}\left|\Omega_{n}(b, \infty)\right|^{1-\frac{\tau}{\hat{\tau}}} \\
& \rightarrow 0
\end{aligned}
$$

as $b \rightarrow \infty$ uniformly in $n$. Using (3.11) again, for any fixed $0<a<b$,

$$
\int_{\Omega_{n}(a, b)}\left|v_{n}\right|^{2} d x=\frac{1}{\left\|u_{n}\right\|^{2}} \int_{\Omega_{n}(a, b)}\left|u_{n}\right|^{2} d x \leq \frac{c_{2}}{C_{a}^{b}\left\|u_{n}\right\|^{2}} \rightarrow 0
$$

as $n \rightarrow \infty$. Let $0<\epsilon<\frac{b_{1,1}}{8}$, by $\left(W_{1}\right)$, there exists $a_{\epsilon}>0$ such that

$$
\left|W_{u}(x, u)\right| \leq \frac{\epsilon}{\gamma_{2}}|u|, \quad \text { for all }|u| \leq a_{\epsilon} .
$$


Consequently,

$$
\int_{\Omega_{n}\left(0, a_{\epsilon}\right)} \frac{W_{u}\left(x, u_{n}\right)}{\left|u_{n}\right|}\left|v_{n}\right|^{2} d x \leq \int_{\Omega_{n}\left(0, a_{\epsilon}\right)} \frac{\epsilon}{\gamma_{2}}\left|v_{n}\right|^{2} d x \leq \frac{\epsilon}{\gamma_{2}}\left\|v_{n}\right\|_{2}^{2} \leq \epsilon
$$

for all $n$. By $\left(W_{3}\right)$ and (3.12), we can take large $b_{\epsilon} \geq r_{0}$ so that

$$
\begin{aligned}
\int_{\Omega_{n}\left(b_{\epsilon}, \infty\right)} \frac{W_{u}\left(x, u_{n}\right)}{\left|u_{n}\right|}\left|v_{n}\right|^{2} d x & \leq\left(\int_{\Omega_{n}\left(b_{\epsilon}, \infty\right)} \frac{\left|W_{u}\left(x, u_{n}\right)\right|^{\sigma}}{\left|u_{n}\right|^{\sigma}} d x\right)^{\frac{1}{\sigma}}\left(\int_{\Omega_{n}\left(b_{\epsilon}, \infty\right)}\left|v_{n}\right|^{2 \sigma^{\prime}} d x\right)^{\frac{2}{2 \sigma^{\prime}}} \\
& \leq\left(\int_{\Omega} c_{0} \tilde{W}\left(x, u_{n}\right) d x\right)^{\frac{1}{\sigma}}\left(\int_{\Omega_{n}\left(b_{\epsilon}, \infty\right)}\left|v_{n}\right|^{\tau} d x\right)^{\frac{2}{\tau}}
\end{aligned}
$$

Hence combining (3.11), (3.12) and (3.14), there is $n_{0}$ such that

$$
\int_{\Omega_{n}\left(b_{\epsilon}, \infty\right)} \frac{\left|W_{u}\left(x, u_{n}\right)\right|\left|v_{n}\right|^{2}}{\left|u_{n}\right|} d x \leq\left(c_{2} c_{0}\right)^{\frac{1}{\sigma}}\left(\int_{\Omega_{n}\left(b_{\epsilon}, \infty\right)}\left|v_{n}\right|^{\tau} d x\right)^{\frac{2}{\tau}}<\epsilon
$$

for $n \geq n_{0}$. Note that there is $\gamma=\gamma(\epsilon)>0$ independent of $n$ such that

$$
\left|W_{u}\left(x, u_{n}\right)\right| \leq \gamma\left|u_{n}\right| \quad \text { for } x \in \Omega_{n}\left(a_{\epsilon}, b_{\epsilon}\right)
$$

By (3.12)

$$
\int_{\Omega_{n}\left(a_{\epsilon}, b_{\epsilon}\right)} \frac{\left|W_{u}\left(x, u_{n}\right)\right|\left|v_{n}\right|^{2}}{\left|u_{n}\right|} d x \leq \gamma \int_{\Omega_{n}\left(a_{\epsilon}, b_{\epsilon}\right)}\left|v_{n}\right|^{2} d x<\epsilon
$$

for all $n \geq n_{0}$. Therefore, combining (3.14)-(3.16), we obtain for $n \geq n_{0}$

$$
\int_{\Omega} \frac{\left|W_{u}\left(x, u_{n}\right)\right|\left|v_{n}\right|^{2}}{\left|u_{n}\right|} d x \leq 3 \epsilon<\frac{b_{1,1}}{2}-\epsilon
$$

which contradicts (3.10). Hence $\left\{u_{n}\right\}$ is bounded in $H$. Going if necessary to a subsequence, we assume that

$$
u_{n} \rightarrow u \quad \text { in } H \text { for some } u \in H \text {, }
$$

which implies $u_{n} \rightarrow u$ a.e. in $\Omega$, because the imbedding $H_{0}^{1}(\Omega) \hookrightarrow L^{2}(\Omega)$ is compact. Hence we have $\left\|u_{n}-u\right\|_{2} \rightarrow 0$ and $\left|\left(\Phi^{\prime}\left(u_{n}\right)-\Phi^{\prime}(u)\right)\left(u_{n}-u\right)\right| \rightarrow 0$. Using the Hölder inequality

$$
\begin{aligned}
& \left|\int_{\Omega}\left(f\left(x, u_{n}(x)\right)-f(x, u(x))\right)\left(u_{n}(x)-u(x)\right) d x\right| \\
& \quad \leq\left(\int_{\Omega}\left|f\left(x, u_{n}(x)\right)-f(x, u(x))\right|^{q}\right)^{\frac{1}{q}}\left(\int_{\Omega}\left|u_{n}(x)-u(x)\right|^{p}\right)^{\frac{1}{p}}
\end{aligned}
$$

$\left(\frac{1}{p}+\frac{1}{q}=1\right)$ for $u_{n} \rightarrow u$ in $L^{p}(\Omega)$, and by $\left(K_{1}\right),\left(K_{2}\right),\left(W_{1}\right),\left(W_{3}\right)$ we have

$$
|f(x, u)| \leq c\left(1+|u|^{p-1}\right)=c\left(1+|u|^{\frac{p}{q}}\right) .
$$


Then, by Lemma 2.2 , we have $f\left(x, u_{n}(x)\right) \rightarrow f(x, u(x))$ in $L^{q}(\Omega)$. Thus

$$
\int_{\Omega}\left(f\left(x, u_{n}(x)\right)-f(x, u(x))\right)\left(u_{n}(x)-u(x)\right) d x \rightarrow 0
$$

as $n \rightarrow+\infty$. Moreover, a straightforward computation shows that

$$
\left(\Phi^{\prime}\left(u_{n}\right)-\Phi^{\prime}(u)\right)\left(u_{n}-u\right)=\left\|\nabla\left(u_{n}-u\right)\right\|_{2}^{2}-\int_{\Omega}\left(f\left(x, u_{n}(x)\right)-f(x, u(x))\right)\left(u_{n}(x)-u(x)\right) d x
$$

it is clear that

$$
\left\|\nabla\left(u_{n}-u\right)\right\|_{2}^{2} \rightarrow 0
$$

Finally,

$$
\left\|u_{n}-u\right\| \rightarrow 0 \text { in } H
$$

This completes the proof.

Now, we are ready to prove Theorem 1.1.

We will obtain a critical point of $\Phi_{\lambda}$ by the use of a standard version of the Mountain Pass Lemma (see [3]). It provides the minimax characterization for the critical value which is important for what follows. Therefore, we state this lemma precisely.

Lemma 3.4 (see [3]) Let $H$ be a real Banach space and $\Phi_{\lambda}: H \rightarrow R^{1}$ be a $C^{1}$-smooth functional. If $\Phi$ satisfies the following conditions:

(i) $\Phi(0)=0$,

(ii) every sequence $\left\{u_{n}\right\}_{n \in N}$ in $H$ such that $\left\{\Phi\left(u_{n}\right)\right\}_{n \in N}$ is bounded in $R^{1}$ and $\Phi^{\prime}\left(u_{n}\right) \rightarrow 0$ in $H^{*}$ as $n \rightarrow+\infty$, contains a convergent subsequence ((PS) condition),

(iii) there are constants $\rho, \alpha>0$ such that $\Phi_{\mid \partial B_{\rho}(0)} \geq \alpha$,

(iv) there is a constant $e \in H \backslash \overline{B_{\rho}(0)}$ such that $\Phi(e) \leq 0$, where $B_{\rho}(0)$ is an open ball in $H$ of radius $\rho$ centered at 0 , then $\Phi$ possesses a critical value $c \geq \alpha$ given by

$$
c=\inf _{g \in \Gamma} \max _{s \in[0,1]} \Phi(g(s))
$$

where

$$
\Gamma=\{g \in C([0,1], H)\}: g(0)=0, g(1)=e\}
$$

Now we are ready to give the proofs of Theorem 1.1.

Proof of Theorem 1.1 Under conditions $\left(F_{1}\right),\left(K_{1}\right),\left(K_{2}\right),\left(W_{1}\right)-\left(W_{3}\right)$, as shown in [9], a deformation lemma can be proved with the $(C)$ condition, replacing the usual PalaisSmale condition, and it turns out that the Mountain Pass Theorem still holds true. Applying the Mountain Pass Lemma 3.4, $\Phi$ possesses a critical value $c \geq \alpha$ given by $c=$ 
$\inf _{g \in \Gamma} \max _{s \in[0,1]} \Phi(g(s))$. Hence, $u$ is a nontrivial solution of problem (P) satisfying $\Phi(u)=c$, $\Phi^{\prime}(u)=0$. The proof is done.

\section{Competing interests}

The authors declare that they have no competing interests.

\section{Authors' contributions}

The paper is the result of joint work of all authors who contributed equally to the final version of the paper. All authors read and approved the final manuscript.

\section{Acknowledgements}

The authors are grateful to anonymous referees for detailed reading of the manuscript and valuable comments, which helped us improve this work. This work was supported by The National Natural Science Foundation of China (No. 11101237) and SNSFC ZR2012AM006.

Received: 14 May 2012 Accepted: 20 August 2012 Published: 31 August 2012

\section{References}

1. Willem, M: Minimax Theorems. Bikhäuser, Boston (1996)

2. Su, JB, Zhao, L: An elliptic resonance problem with multiple solutions. J. Math. Anal. Appl. 319, 604-616 (2006)

3. Ambrosetti, A, Rabinowitz, PH: Dual variational methods in critical point theory and applications. J. Funct. Anal. 14, 349-381 (1973)

4. Schechter, M, Zou, W: Superlinear problems. Pac. J. Math. 214, 145-160 (2004)

5. Costa, DG, Magalhães, CA: Variational elliptic problems which are nonquadratic at infinity. Nonlinear Anal., Theory Methods Appl. 23, 1401-1412 (1994)

6. Szulkin, A, Zou, W: Homoclinic orbits for asymptotically linear Hamiltonian system. J. Funct. Anal. 187, 25-41 (2001)

7. Jeanjean, L: On the existence of bounded Palais-Smale sequences and application to a Landesman-Lazer type problem set on $R^{N}$. Proc. R. Soc. Edinb., Sect. A, Math. 129, 787-809 (1999)

8. Miyagaki, OH, Souto, MAS: Superlinear problems without Ambrosetti and Rabinowitz growth condition. J. Differ. Equ. 12, 3628-3638 (2008)

9. Schechter, M: Superlinear elliptic boundary value problems. Manuscr. Math. 86, 253-265 (1995)

10. Rabinowitz, PH, Su, JB, Wang, ZQ: Multiple solutions of superlinear elliptic equations. Rendiconti Lincei Matematicae Applicazioni 18, 97-108 (2007)

11. Szulkin, A, Weth, T: Ground state solutions for some indefinite variational problems. J. Funct. Anal. 257, 3802-3822 (2009)

12. Rabinowitz, PH: Minimax Methods in Critical Point Theory with Applications to Differential Equations, CBMS (1986)

13. Izydorek, M, Janczewska, J: Homoclinic solutions for a class of the second order Hamiltonian systems. J. Differ. Equ. 219, 375-389 (2005)

14. Coti-Zelati, V, Rabinowitz, P: Homoclinic type solutions for a semilinear elliptic PDE on $R^{n}$. Commun. Pure Appl. Math. 46, 1217-1269 (1992)

15. Ding, YH, Luan, SX: Multiple solutions for a class of Schrödinger equations. J. Differ. Equ. 207, 423-457 (2004)

16. Shi, SJ, Li, SJ: Existence of solutions for a class of semilinear elliptic equations with the Robin boundary value condition. Nonlinear Anal., Theory Methods Appl. 71, 3292-3298 (2009)

17. Bartsch, T, Ding, YH: On a nonlinear Schrödinger equation with periodic potential. Math. Ann. 313, 15-37 (1999)

18. Li, SJ, Su, JB: Existence of multiple solutions of a two-point boundary value problem at resonance. Nonlinear Anal., Theory Methods Appl. 10, 123-135 (1997)

19. Ding, YH, Lee, C: Multiple solutions of Schrödinger equations with indefinite linear part and super or asymptotically linear terms. J. Differ. Equ. 222, 137-163 (2006)

20. Zhao, FK, Chen, J, Yang, MB: A periodic solution for a second-order asymptotically linear Hamiltonian system. Nonlinear Anal., Theory Methods Appl. 70, 4021-4026 (2009)

doi:10.1186/1687-2770-2012-97

Cite this article as: Mao et al.: Existence of solutions of elliptic boundary value problems with mixed type nonlinearities. Boundary Value Problems 2012 2012:97. 